\title{
Management of Ruptured and Rapidly Progressive Mycotic Cerebral Aneurysms in the Setting of Unilateral Carotid Occlusion and Endocarditis with Valve Failure
}

\author{
Robert C. Rennert ${ }^{1} \quad$ David R. Santiago-Dieppa ${ }^{1} \quad$ J. Scott Pannell ${ }^{1} \quad$ Alexander A. Khalessi ${ }^{1}$ \\ ${ }^{1}$ Division of Neurosurgery, University of California, San Diego, San \\ Diego, California, United States \\ J Neurol Surg Rep 2015;76:e222-e226.

\begin{abstract}
Address for correspondence Alexander A. Khalessi, MD, MS, FAANS, FAHA, Division of Neurosurgery, Department of Surgery and Neurosurgery, University of California, San Diego, 200 West Arbor Drive, San Diego, CA 92103, United States (e-mail: akhalessi@ucsd.edu).
\end{abstract}

\author{
Abstract \\ Keywords \\ - mycotic aneurysm \\ - middle cerebral artery \\ bifurcation aneurysm \\ - anterior \\ communicating \\ artery aneurysm \\ - endovascular \\ treatment \\ - microneurosurgical \\ treatment
}

Mycotic cerebral aneurysms can present unique neurosurgical challenges. We report a patient with left carotid occlusions, a ruptured left middle cerebral artery mycotic aneurysm, and a rapidly appearing unruptured left anterior cerebral artery/anterior communicating artery (ACA/ACom) mycotic aneurysm in the setting of mitral valve endocarditis with a perivalvular leak and evolving congestive heart failure. Following medical stabilization and antibiotic administration, a combined endovascular (with contralateral access via the ACom) and open surgical approach was used to selectively secure both aneurysms with preservation of distal flow, allowing lifesaving cardiac valve replacement. This case illustrates the therapeutic complexity of mycotic cerebral aneurysms, which we discuss in the context of an increasing reliance on endovascular approaches.

\section{Introduction}

Rupture of mycotic cerebral aneurysms is associated with significant morbidity and mortality; however, unlike cerebral aneurysms from noninfectious causes, unruptured mycotic aneurysms can resolve with medical treatment. ${ }^{1,2}$ Management of cerebral mycotic aneurysms requires an understanding of their pathogenesis, natural history, and treatment options. The need for heparinization for cardiac surgery in patients with cardiovascular compromise due to septic endocarditis must also be considered. The following case highlights the benefit of a multimodal therapeutic approach tailored to individual aneurysm morphology.

\section{Case Report}

A 29-year-old man with a history of drug abuse, right parietal abscess status post craniotomy and resection, and endocarditis status post bioprosthetic valve replacement presented to an area hospital with generalized aches and malaise. Outpatient blood culture was positive for $\alpha$ hemolytic streptococcus. Initial workup revealed bioprosthetic mitral valve endocarditis with vegetations. Broad-spectrum antibiotics were started and tailored after repeat blood cultures were positive for Staphylococcus haemolyticus. Neuroimaging for transient aphasia revealed a distal left middle cerebral artery (MCA) aneurysm, occlusion of the left internal carotid artery (ICA), and vasospasm in the bilateral MCAs and basilar artery. The patient was transferred to our institution for further care.

Upon arrival the patient's neurologic examination was significant for $4 / 5$ motor strength on the left hemibody secondary to his old abscess. Magnetic resonance imaging/ magnetic resonance angiography (MRI/MRA) and cerebral angiogram demonstrated a large $(11 \times 9 \mathrm{~mm})$ multilobulated mycotic aneurysm at the left M2-M3 junction involving the origin of both M3 branches and the distal aspect of the M2
License terms

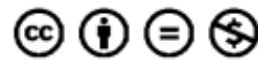

received

January 13, 2015

accepted

May 16, 2015

published online

August 3, 2015
DOI http://dx.doi.org/

10.1055/s-0035-1555748. ISSN 2193-6358. (c) 2015 Georg Thieme Verlag KG
Stuttgart · New York 
branch ( - Fig. 1A, B), with surrounding susceptibility artifact and edema consistent with prior hemorrhage (-Fig. 1C). Complete occlusions of the left ICA and multiple branches of the left external carotid artery were noted. The left MCA and anterior cerebral artery (ACA) distributions filled via the posterior communicating artery and the anterior communicating artery (ACom) and leptomeningeal collaterals.

Intervention was initially deferred due to the potential need for M3 branch sacrifice, with external carotid occlusions preventing bypass. The patient remained a poor surgical candidate due to ongoing treatment for sepsis, vasospasm, and cerebral salt wasting, as well as multiple venous and bilateral arterial thrombi obstructing femoral access. Surveillance computed tomography angiography (CTA) 6 days after admission demonstrated a new $7 \times 7 \mathrm{~mm}$ saccular aneurysm at the junction of the left A1 segment and ACom (-Fig. 2A, B).
The patient concurrently developed high-output congestive heart failure (CHF) due to perivalvular mitral leak, necessitating immediate repair. Given the unsecured, ruptured, and rapidly growing mycotic aneurysms, however, heparinization was felt to be unsafe. A combined approach to secure both cerebral aneurysms was used in preparation for cardiac surgery.

On postadmission day 10, iliac artery embolectomy and permanent coil embolization of the left MCA aneurysm was performed, with the likely chronic left ICA occlusion requiring microcatheterization of the left MCA from the right side via the ACom ( - Fig. 3A-C). A PX SLIM microcatheter (Penumbra Inc., Alameda, California, United States) and Fathom 0.16 guidewire (Boston Scientific, Marlboro, Massachusetts, United States) were used for microcatheterization, with the diameter of the PX SLIM allowing for meaningful angiographic runs. To ensure a high degree of control with coil contouring
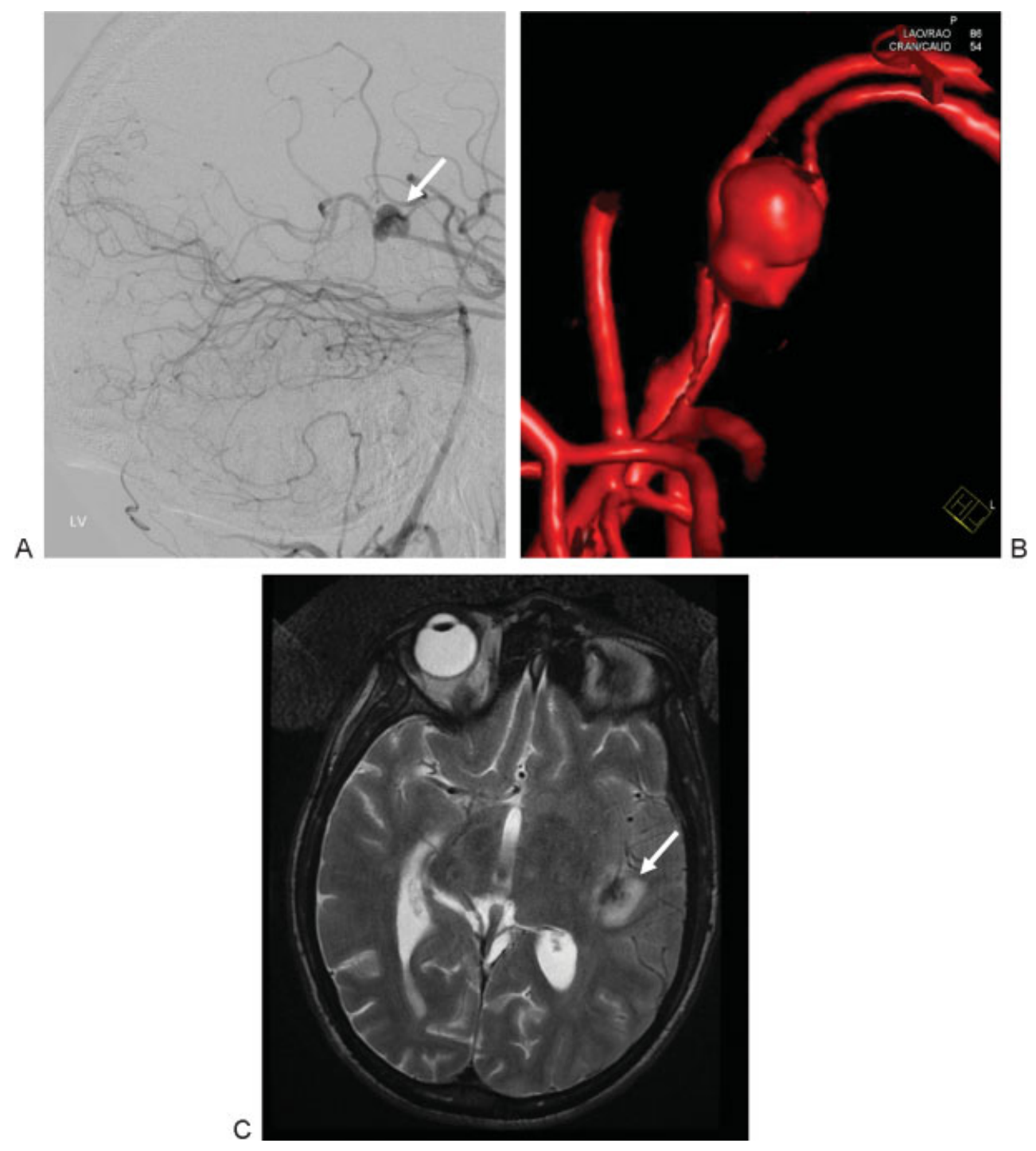

Fig. 1 Previously ruptured distal middle cerebral artery mycotic aneurysm. (A) Lateral left vertebral injection demonstrating an $11 \times 9 \mathrm{~mm}$ multilobulated mycotic aneurysm (white arrow) at the left M2-M3 junction. The left hemisphere relied predominantly on a left posterior communicating artery for collateral supply due to chronic left internal carotid artery occlusion. (B) Three-dimensional reconstruction demonstrating involvement of the bilateral M3 origins as well as the distal aspect of the M2 branch, with proximal stenosis noted. (C) T2-weighted magnetic resonance imaging demonstrating perianeurysmal susceptibility artifact and edema (white arrow) consistent with prior hemorrhage. 

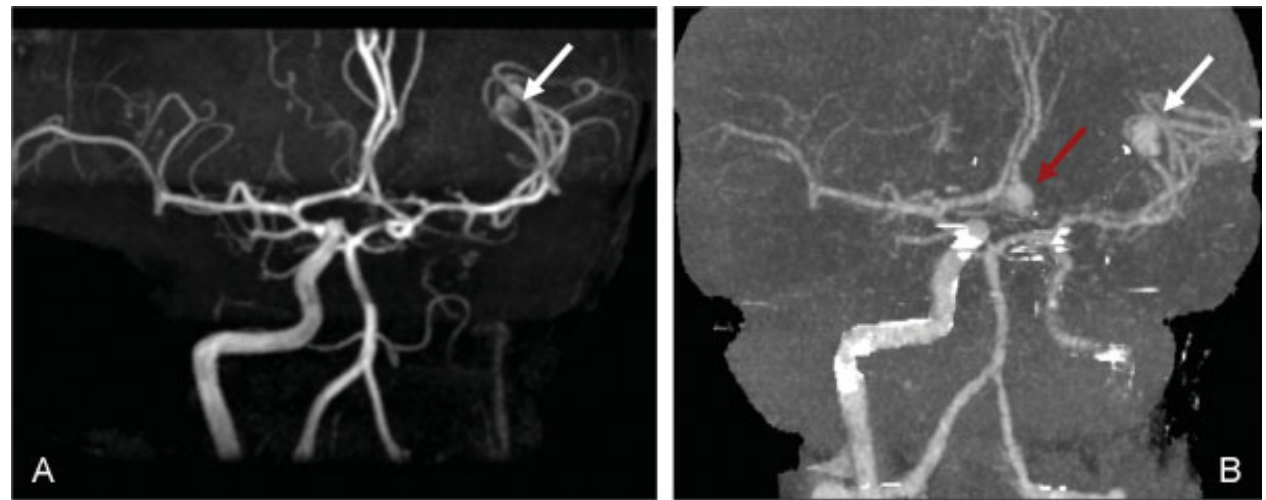

Fig. 2 Rapidly arising anterior cerebral artery/anterior communicating artery (ACA/ACom) mycotic aneurysms. (A) Initial magnetic resonance angiography and (B) computed tomography angiography 6 days later demonstrating a rapidly arising $7 \times 7$ mm saccular aneurysm (red arrow) arising from the junction of the left A1 segment and the ACom that developed despite aggressive broad-spectrum antibiotic administration. The distal left middle cerebral artery aneurysm is readily visualized on both scans (white arrows).
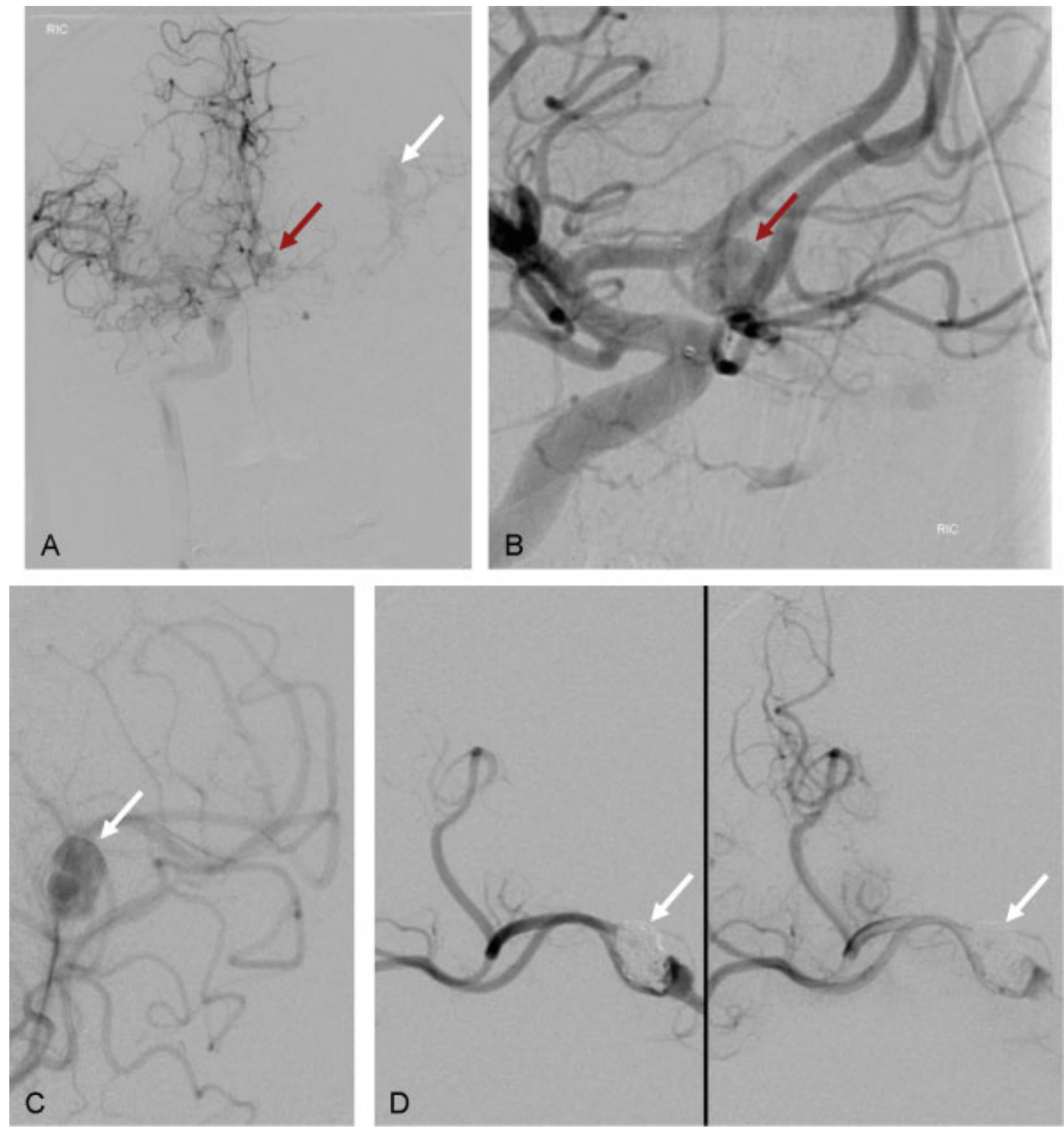

Fig. 3 Therapeutic approach to multifocal mycotic aneurysms with limited arterial access. (A) Right internal carotid artery (ICA) anteroposterior angiogram demonstrating contralateral middle cerebral artery (MCA) filling due to chronic left ICA occlusion, and left anterior cerebral artery/ anterior communicating artery (ACA/ACom) (red arrow) and distal middle cerebral artery (MCA) (white arrow) mycotic aneurysms. (B) Redemonstration of the left ACA/ACom aneurysm (red arrow) following right ICA injection. This aneurysm could not be accessed endovascularly and was subsequently clipped via a minimally invasive right supraorbital craniotomy. (C) Left MCA lateral microcatheter angiogram performed before coil embolization of the left MCA aneurysm (white arrow), accessed after crossing the ACom aneurysm due to rupture status and left ICA occlusion. (D) Multiple views of a selective left MCA angiogram performed after coil embolization of the left MCA aneurysm via microcatheter placed across the ACom, demonstrating patency of all left M3 vessels and a Raymond 0 occlusion of the aneurysm (white arrow). 
and preserve distal branch flow, three coils of varying lengths and stiffness were used for embolization $(9 \mathrm{~mm} \times 25 \mathrm{~cm}$ and $6 \mathrm{~mm} \times 15 \mathrm{~cm}$ complex soft, and $4 \mathrm{~mm} \times 8 \mathrm{~cm}$ complex extra soft Penumbra 400 coils; Penumbra Inc.). Subsequent angiography demonstrated patency of all M3 vessels and complete aneurysmal occlusion, a Raymond 0 result (-Fig. 3D). The left ACA/ACom aneurysm could not be accessed via microcatheterization without parent artery compromise due to diffuse disease of the A1/A2 junction and the ACom. Four days later, the saccular ACA/ACom aneurysm was visualized and clipped via a minimally invasive right supraorbital craniotomy.

The patient tolerated these procedures well and received a mitral valve replacement 6 days after aneurysm clipping. The patient's CHF completely resolved, and he was discharged 2 days later to a skilled nursing facility, with his neurologic examination at baseline from admission.

\section{Discussion}

Mycotic cerebral aneurysms comprise 1 to $5 \%$ of all intracranial aneurysms. They are caused by septic microemboli to the vasa vasorum or direct bacterial migration from an occluding septic embolus, ${ }^{1}$ with the resulting inflammation causing localized vessel wall weakness. The most common source of emboli is bacterial endocarditis, as seen in this case. The most common pathogens are Streptococcus and Staphylococcus species. ${ }^{1,3}$ Mycotic aneurysms are most common within cerebral arteries, specifically at distal branch points of the MCA, ${ }^{1,4}$ and they often occur at multiple sites.

Cerebral arterial imaging is recommended for infectious endocarditis only in the presence of neurologic symptoms. ${ }^{4}$ Visualization of an aneurysm (typically fusiform or saccular, with multiplicity, eccentricity, and/or a multilobed structure with surrounding inflammation) with positive blood cultures and an embolic source are often cited criteria for diagnosis. ${ }^{3,5}$ CT-CTA and MRA are typical first-line imaging tests, yet digital subtraction angiography remains the gold standard. . $^{3,4}$ In this case, the patient had vegetative endocarditis with positive blood cultures. Cerebral imaging was obtained for transient aphasia, revealing a single eccentric saccular aneurysm, with subsequent growth of a second, more proximal aneurysm in the face of optimized antibiotic therapy.

The preferred treatment for mycotic cerebral aneurysms lacks level 1 evidence and is confounded by the potential for unruptured aneurysms to resolve with antibiotic therapy. Stable unruptured mycotic aneurysms were traditionally managed with antibiotics alone; ruptured or enlarging unruptured aneurysms were thought to require intervention. ${ }^{1,6}$ Recently proposed guidelines advocate for intervention for all but the highest risk mycotic aneurysms, however, regardless of rupture status. ${ }^{3}$ In this patient, both aneurysms required intervention given their previous rupture and rapid growth, respectively, as well as the need for anticoagulation for mitral valve replacement.

When deciding between an open and endovascular approach, standard considerations in the treatment of cerebral aneurysms are relevant. Older patient age, morbidity, and surgically inaccessible sites favor endovascular techniques, whereas younger age, morbidity, and the presence of hematoma with mass effect favor surgical resection. Surgical management of mycotic aneurysms is nonetheless complicated by the friability of infected vessels, and it may increase the complication rate of subsequent cardiac valve replacement that requires heparinization and anticoagulation. ${ }^{3}$

The goal of either approach is to secure the at-risk aneurysm and prevent strokes. Decisions regarding primary or secondary preservation of distal flow are based on individual aneurysm characteristics and the presence of eloquent tissue distally. ${ }^{6}$ Endovascular options include selective or parent artery coil embolization. Surgical options include clipping and ligation/excision alone or with bypass. Although there is no definitive answer regarding the timing of intervention, prior antibiotic treatment is thought to decrease interventional risk by allowing the aneurysm to become more fibrotic. $^{3}$ This potential must be balanced with the ongoing interval hemorrhage risk from an unsecured aneurysm.

In the present case, early endovascular intervention of the MCA saccular aneurysm was not performed because selective embolization was not possible in the setting of acute infection, and sacrifice of the two M3 branches with parent vessel occlusion was unpalatable. Occlusion of extracranial donor bypass vessels prevented early surgical intervention with preservation of distal perfusion via extracranial to intracranial bypass, and the long axial nature of the aneurysm made in situ end-to-end anastomosis challenging. Concerns over vessel friability in the setting of local infection further dampened enthusiasm for other surgical approaches, such as MCA-MCA bypass. During the patient's subsequent sepsis management, bilateral occlusion of the iliac arteries limited endovascular access, and surveillance imaging identified a new saccular left ACA aneurysm. Following medical stabilization and antibiotic treatment, iliac artery embolectomy and selective endovascular embolization of both aneurysms was attempted via a contralateral ACom approach due to chronic left ICA occlusion. This maneuver limited the vascular territories placed at risk from catheter manipulation to those with existing disease. Nonetheless, only the MCA aneurysm could be safely secured with this approach. The ACom aneurysm was then surgically clipped through a small supraorbital craniotomy, minimizing risk for subsequent cardiac valve replacement.

\section{Conclusion}

Although endovascular techniques have improved our ability to manage mycotic cerebral aneurysms, their heterogeneous nature may still necessitate a combined approach. Ultimately, aneurysm rupture status, technical risks of neurosurgical management, response to antibiotics, and urgency of cardiac surgery requiring heparinization will be decisive elements of a treatment plan.

\section{Highlights}

- Mycotic cerebral aneurysms are difficult to manage and require individualized treatment. 
- Endovascular approaches have enabled more aggressive management regardless of rupture status.

- Patient comorbidities and the heterogeneous nature of mycotic cerebral aneurysms may require creative therapeutic approaches to enable critical cardiac interventions.

\section{Disclosures/Consent}

Alexander A. Khalessi has previously received competitive grants from Covidien Ltd. and Penumbra Inc., and he holds consulting arrangements for physician training with Stryker Neurovascular, Covidien Ltd., and Penumbra Inc. He has no direct financial interests related to this work. The other authors have nothing to disclose. Institutional review board approval was not required for preparation of the present report. Informed consent from the patient was obtained before commencement of all procedures and permitted publication of this report.

\section{References}

1 Peters PJ, Harrison T, Lennox JL. A dangerous dilemma: management of infectious intracranial aneurysms complicating endocarditis. Lancet Infect Dis 2006;6(11):742-748
2 Monsuez JJ, Vittecoq D, Rosenbaum A, et al. Prognosis of ruptured intracranial mycotic aneurysms: a review of 12 cases. Eur Heart J 1989;10(9):821-825

3 Zanaty M, Chalouhi N, Starke RM, et al. Endovascular treatment of cerebral mycotic aneurysm: a review of the literature and single center experience. Biomed Res Int 2013;2013:151643

4 Baddour LM, Wilson WR, Bayer AS, et al; Committee on Rheumatic Fever, Endocarditis, and Kawasaki Disease; Council on Cardiovascular Disease in the Young; Councils on Clinical Cardiology, Stroke, and Cardiovascular Surgery and Anesthesia; American Heart Association; Infectious Diseases Society of America. Infective endocarditis: diagnosis, antimicrobial therapy, and management of complications: a statement for healthcare professionals from the Committee on Rheumatic Fever, Endocarditis, and Kawasaki Disease, Council on Cardiovascular Disease in the Young, and the Councils on Clinical Cardiology, Stroke, and Cardiovascular Surgery and Anesthesia, American Heart Association: endorsed by the Infectious Diseases Society of America. Circulation 2005;111(23): e394-e434

5 Kannoth S, Thomas SV, Nair S, Sarma PS. Proposed diagnostic criteria for intracranial infectious aneurysms. J Neurol Neurosurg Psychiatry 2008;79(8):943-946

6 Chun JY, Smith W, Halbach VV, Higashida RT, Wilson CB, Lawton MT. Current multimodality management of infectious intracranial aneurysms. Neurosurgery 2001;48(6):1203-1213; discussion 1213-1214 